\title{
ON SUBCLASSES OF CLOSE-TO-CONVEX FUNCTIONS OF HIGHER ORDER
}

\author{
KHALIDA INAYAT NOOR \\ Mathemat Ics Departinent \\ P.0. Box 2455, King Saud University \\ Riyadh 11451, Saudi Arabla \\ (Received October 30, 1990 and in revised form October 21, 1991)
}

ABSTRACT. The classes $T_{k}(\rho), 0 \leqslant \rho<1, k>2$, of analytic functions, using the class $v_{k}(\rho)$ of functions of bounded boundary rotation, are defined and it is shown that the functions in these classes are close-toconvex of higher order. Covering theorem, arc-length result and some radii problems are solved. We also discuss some properties of the class $v_{k}(p)$ including distortion and coefficient results.

1980 AMS SUBJECT CLASSIFICATION. 30C45.

KEY WORDS AND PHRASES: Analytic functions, close-to-convex, univalent, bounded boundary rotation, coefficient, positive real part.

1. THE CLASS $\mathbf{P}_{\mathbf{k}}(\rho)$

Let $P_{k}(\rho)$ be the class of functions $p(z)$ analytic in the unit disc $E=$ $\{z:|z|<1\}$ satisfying the properties $p(0)=1$ and

$$
\int_{0}^{2 \pi}\left|\frac{\operatorname{Re} \rho(z)-\rho}{1-\rho}\right| d \theta<k \pi,
$$

where $z=r e^{i 0}, k>2$ and $0<p<1$. This class has been introduced in [1]. We note that, for $\rho=0$, we obtain the class $P_{k}$ defined by Pinchuk [2] and for $\rho$ $=0, k=2$, we have the class $P$ of functions with positive réal part. The case $k=2$ gives us the class $P(\rho)$ of functions with positive real part greater than $\rho$.

Also we can write

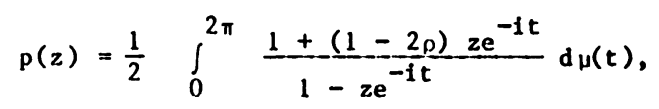

where $\mu(t)$ is a function with bounded variation on $[0,2 \pi]$ such that

and

$$
\left.\begin{array}{l}
\int_{0}^{2 \pi} d \mu(t)=2 \\
\int_{0}^{2 \pi}|d \mu(t)|<k
\end{array}\right\}
$$

From (1.1), we have the following. 
THEOREN 1.1. Let $p \in \mathrm{P}_{\mathrm{k}}(0)$. Then

$$
p(z)=\left(\frac{k}{4}+\frac{1}{2}\right) p_{1}(z)-\left(\frac{k}{4}-\frac{1}{2}\right) p_{2}(z),
$$

whee $p_{i} F(n), i=1,2$.

We now prove.

THEOREM 1.2. The class $P_{k}(n)$ is a convex set.

PROOF. Let $H_{1}, H_{2}$ \& $P_{k}(\rho)$. We shall show that, for $\alpha, \beta>0$

$$
H(z)=\frac{1}{\alpha+\beta}\left[\alpha H_{1}(z)+\beta H_{2}(z)\right]
$$

belongs to $P_{k}(n)$.

From Theorem 1.1 , we can write

$$
\begin{aligned}
H(z)=\frac{1}{\alpha+\beta}\{\alpha & \left.\left(\frac{k}{4}+\frac{1}{2}\right) p_{1}(z)-\left(\frac{k}{4}-\frac{1}{2}\right) p_{2}(z)\right\} \\
& \left.+\beta\left\{\left(\frac{k}{4},+\frac{1}{2}\right) p_{3}(z)-\left(\frac{k}{4}-\frac{1}{2}\right) p_{4}(z)\right\}\right],
\end{aligned}
$$

where $p_{i} \in P(\rho), i=1,2,3,4$.

we have

$$
\text { Now, writing } p_{i}(z)=(1-n) h_{i}(z)+n, i=1,2,3,4 \text {, see }[3] \text {, }
$$

$$
\begin{aligned}
\frac{H(z)-\rho}{1-\rho} & =\left(\frac{k}{4}+\frac{1}{2}\right)\left\{\frac{1}{\alpha+\beta}\left(\alpha h_{1}(z)+R h_{3}(z)\right)\right\}-\left(\frac{k}{4}-\frac{1}{2}\right)\left\{\frac{1}{\alpha+\beta}\left(\alpha h_{2}(z)+\rho h_{4}(z)\right)\right\} \\
& =\left(\frac{k}{4}+\frac{1}{2}\right) f_{1}(z)-\left(\frac{k}{4}-\frac{1}{2}\right) f_{2}(z),
\end{aligned}
$$

where $f_{1}$ and $f_{2} E P$, since $P$ is a convex set, see [2] and this gives us the required result.

THEOREM 1.3. Let $p \in P_{k}(\rho)$ and be given by $\dot{p}(z)=1+\sum_{n=1}^{\infty} c_{n} z^{n}$. Then

(i) $\frac{1}{2 \pi} \int_{0}^{2 \pi}\left|p\left(r e^{i \theta}\right)\right|^{2} d \theta<\frac{1+\left[k^{2}(1-\rho)^{2}-1\right] r^{2}}{1-r^{2}}$

and

(ii) $\frac{1}{2 \pi} \int_{0}^{2 \pi}\left|p^{\prime}\left(r e^{i \theta}\right)\right| d \theta<\frac{k(1-0)}{1-r^{2}}$

PROOF. (i) Using Parseval's identity, we have

$$
\begin{aligned}
\frac{1}{2 \pi} \int_{0}^{2 \pi}\left|\mathrm{p}\left(\mathrm{re}^{1 \theta}\right)\right| \mathrm{d} \theta & =\sum_{n=0}^{\infty}\left|\mathrm{c}_{n}\right|^{2} \mathrm{r}^{2 \mathrm{n}} \\
& =1+k^{2}(1-\rho)^{2} \sum_{n=1}^{\infty} \mathrm{r}^{2 n}=\frac{1+\left[k^{2}(1-\rho)^{2}-1\right] \mathrm{r}^{2}}{\left(1-\mathrm{r}^{2}\right)},
\end{aligned}
$$

where we have used an easily established sharp result $\left|c_{n}\right|<k(1-\rho)$, for all $n>1$. 
(ii) By using Theorem 1.1, we can write

$$
p(z)-\rho=\left(\frac{k}{4}+\frac{1}{2}\right)(1-p) h_{1}(z)-\left(\frac{k}{4}-\frac{1}{2}\right)(1-p) h_{2}(z),
$$

where $h_{1}, h_{2} \in P$.

Therefore,

$$
p^{\prime}(z)=\left(\frac{k}{4}+\frac{1}{2}\right)(1-0) h_{1}^{\prime}(z)-\left(\frac{k}{4}-\frac{1}{2}\right)(1-p) h_{2}^{\prime}(z)
$$

Now, for all heP, we have

$$
h^{\prime}(z)=\frac{2 w^{\prime}(z)}{(1+w(z))^{2}}
$$

where $w(z)$ is a Schwarz function [3], and

$$
\begin{aligned}
\frac{1}{2 \pi} \int_{0}^{2 \pi}\left|h^{\prime}\left(r e^{i 0}\right)\right| d \theta & =\frac{1}{2 \pi} \int_{0}^{2 \pi} \frac{2\left|w^{\prime}\left(r e^{i \theta}\right)\right|}{\left|1+w\left(r e^{i \theta}\right)\right|^{2}} d \theta \\
& <\frac{2}{1-r^{2}}
\end{aligned}
$$

Hence, from (1.4) and (1.5), we have

$$
\frac{1}{2 \pi} \int_{0}^{2 \pi}\left|p^{\prime}\left(r e^{i \theta}\right)\right| d \theta<\frac{k(1-\rho)}{1-r^{2}}
$$

which is the required result.

From Theorem 1.1 and the properties of the class $P(p)$, we immediately have the following.

THEOREM 1.4. Let $p \in P_{k}(p)$. Then

$\frac{1-k(1-\rho) r+(1-2 \rho) r^{2}}{1-r^{2}}<\operatorname{Re} p(z)<\frac{1+k(1-\rho) r+(1-2 \rho) r^{2}}{1-r^{2}}$

THEOREM 1.5. Let $\operatorname{peP}_{k}(\rho)$. Then $p \in P$ for $|z|<r_{0}$, where $r_{0}$ is given by

$$
r_{0}=2 /\left[k(1-\rho)+\sqrt{k^{2}(1-\rho)^{2}-4(1-2 \rho)}\right], \rho \neq \frac{1}{2}
$$

When $\rho=0$, we obtain the results proved in [2].

\section{THE CLASS $\mathbf{v}_{k}(p)$}

DEFINITION 2.1. Let $v_{k}(p)$ denote the class of analytic and locally univalent functions $f$ in $E$ with normalization $f(0)=0, f^{\prime}(0)=1$ and satisfying the condition

$$
\frac{\left(z f^{\prime}(z)\right)^{\prime}}{f^{\prime}(z)} \in P_{k}(\rho), \quad 0<\rho<1, \quad k>2
$$

When $\rho=0$, we obtain the class $v_{k}$ of functions with bounded boundary rotation. The class $V_{k}(\rho)$ also generalizes the class $C(\rho)$ of convex functions of order $\rho$.

It can easily be seen [1] that $f \in V_{k}(\rho)$ if and only if there 
exists $F \in V_{k}$ such that

$$
f^{\prime}(z)=\left(F^{\prime}(z)\right)^{l-\rho}
$$

In the following, we will study the distortion theorems for the class $v_{k}(\rho)$. We will use the hypergeometric functions

$$
\begin{aligned}
G(a, b ; c, z) & =\frac{\Gamma(c)}{\Gamma(a) \Gamma(b)} \sum_{n=0}^{\infty} \frac{\Gamma(a+n)}{\Gamma(c+n)} \frac{\Gamma^{2}(b+n)}{n !} \frac{z^{n}}{n !} \\
& =\frac{\Gamma(c)}{\Gamma(a) \Gamma(c-a)} \int_{0}^{1} u^{a-1}(1-u)^{c-a-1}(1-z u)^{-b} d u,
\end{aligned}
$$

where $\operatorname{Re} a>0$ and $\operatorname{Re}(c-a)>0$. These functions are analytic for $z \varepsilon E[4]$. In addition, we define the functions

and

$$
M_{1}(a, b ; c, r)=\frac{2^{b-1}}{a}\left[G(a, b ; c,-1)-r_{1}^{-a} G\left(a, b ; c,-r_{1}^{-1}\right)\right]
$$$$
M_{2}(a, b ; c, r)=\frac{2^{b-1}}{a}\left[G(a, b ; c,-1)-r_{1}^{a} G\left(a, b ; c,-r_{1}\right)\right] \text {, }
$$

where $\quad r_{1}=\frac{1-r}{1+r}$

THEOREM 2.1. Let. $\mathrm{f} \varepsilon \mathrm{V}_{\mathrm{k}}(\rho)$. Then, for $|z|=r(0<\mathrm{r}<1)$, we have

$$
M_{2}(a, b ; c, r)<|f(z)|<M_{1}(a, b ; c, r),
$$

where

$$
\left.\begin{array}{l}
a=\left(\frac{k}{2}-1\right)(1-\rho)+1, \\
b=2 \rho \\
c=\left(\frac{k}{2}-1\right)(1-\rho)+2
\end{array}\right\}
$$

and $M_{1}, M_{2}$ are as defined in (2.2).

This result is sharp.

PROOF. Using (2.1) and the well-known bounds for $\left|F^{\prime}(z)\right|$ with $F \in V_{k}$, see [2], we have

$$
\frac{(1-|z|)^{\left(\frac{k}{2}-1\right)(1-\rho)}}{(1+|z|)^{\left.\frac{k}{2}+1\right)(1-\rho)}}<\left|f^{\prime}(z)\right|<\frac{(1+|z|)^{\left(\frac{k}{2}-1\right)(1-\rho)}}{(1-|z|)^{\left(\frac{k}{2}+1\right)(1-\rho)}}
$$

Let $d_{r}$ denote the radius of the largest schlicht disk centered at the origin contained in the image of $|z|<r$ under $f(z)$. Then there is a point $z_{0},\left|z_{0}\right|=r$, such that $\left|f\left(z_{0}\right)\right|=d_{r}$. The ray from 0 to $f\left(z_{0}\right) 11 e s$ entirely in the image of $E$ and the inverse image of this ray is a curve in $|z|<r$.

Thus

$$
d_{r}=\left|f\left(z_{0}\right)\right|=\int_{C}\left|f^{\prime}(z)\right||d z|
$$




$$
\begin{aligned}
& >\int_{C} \frac{(1-|z|)^{\left(\frac{k}{2}-1\right)(1-0)}}{(1+|z|)^{\left(\frac{k}{2}+1\right)(1-p)}|d z|} \\
& >\int_{0}^{|z|} \frac{(1-t)}{(1+t)\left(\frac{k}{2}-1\right)(1-0)} \frac{\left(\frac{k}{2}+1\right)(1-0)}{(1+t)} \\
& =\int_{0}^{|z|}\left(\frac{1-t}{1+t}\right)^{\left(\frac{k}{2}-1\right)(1-0)} \frac{d t}{(1+t)^{2(i-p)}}
\end{aligned}
$$

Let $\frac{1-t}{1+t}=\xi$. Then $\frac{-2}{(1+t)^{2}} d t=d \xi$.

So

$$
\begin{aligned}
\left|f\left(z_{0}\right)\right|>2^{2 \rho-1} & \mid \int_{0}^{1} \xi^{\left(\frac{k}{2}-1\right)(1-\rho)}(1+\xi)^{-2 \rho} d \xi \\
& \left.-\int_{0}^{\frac{1-|z|}{1+|z|}\left(\frac{k}{2}-1\right)(1-\rho)}(1+\xi)^{-2 \rho} d \xi\right\}
\end{aligned}
$$

Put $\frac{1-|z|}{1+|z|}=\frac{1-r}{1+r}=r_{1}$ and $\xi=r_{1} u$.

This gives

$$
\begin{aligned}
\left|f\left(z_{0}\right)\right| & >\frac{2^{b-1}}{a}\left\{G(a, b ; c,-1)-r_{1}^{a} G\left(a, b ; c,-r_{1}\right)\right\} \\
& =M_{2}(a, b, c, r),
\end{aligned}
$$

where $a, b, c$ and $M_{2}$ are respectively defined by (2.4) and (2.2). Similarly we can calculate the lower bound for $|f(z)|$ and this establishes our result.

Equality is attained in (2.3) for the function $f_{0} \varepsilon v_{k}(p)$ defined by

$$
f_{0}^{\prime}(z)=\frac{\left(1+\delta_{1} z\right)^{\left(\frac{k}{2}-1\right)(1-p)}}{\left(1-\delta_{2} z\right)^{\left(\frac{k}{2}+1\right)(1-p)}},\left|\delta_{1}\right|=\left|\delta_{2}\right|=1
$$

We now study the behaviour of the integral transform

$$
f_{\alpha}(z)=\int_{0}^{z}\left(f^{\prime}(\xi)\right)^{\alpha} d \xi
$$

for $f \in V_{k}(\rho)$

This problem has been studied for the class of univalent normalized functions in $E$ and for the close-to-convex functions, see [3]. We have 
THEOREM 2.2. Let $f \in V_{k}(p), 0<\rho<1, k>2$ and let $\alpha, 0<\alpha<1$ be given. Then $f_{\alpha} \neq V_{m}$ for $m \leqslant\{\alpha(1-\rho)(k-2)+2\}$.

PROOF. From (2.1), we have

Now

$$
f^{\prime}(z)=\left(F^{\prime}(z)\right)^{1-\rho}, \quad F \in V_{k}
$$

$$
\begin{aligned}
f_{\alpha}^{\prime}(z) & =\left(f^{\prime}(z)\right)^{\alpha}=\left(F^{\prime}(z)\right)^{\alpha(1-\rho)} \\
& =\exp \int_{-\pi}^{\pi}-\log \left(1-\xi e^{-i t}\right) \alpha(1-\rho) \operatorname{dm}(t) \\
& =\exp \int_{-\pi}^{\pi}-\log \left(1-\xi e^{-i t}\right) d \mu(t)
\end{aligned}
$$

where $d \mu(t)=\alpha(1-\rho) d m(t)+[1-\alpha(1-\rho)] \frac{d t}{\pi}$

Also

$$
\int_{-\pi}^{\pi} d \mu(t)=\alpha(1-\rho) \int_{-\pi}^{\pi} d m(t)+\frac{1-\alpha(1-\rho)}{\pi} \int_{-\pi}^{\pi} d t=2,
$$

and

$$
\begin{aligned}
\int_{-\pi}^{\pi}|d \mu(t)| & <\alpha(1-\rho) \int_{-\pi}^{\pi}|d m(t)|+\frac{1-\alpha(1-\rho)}{\pi} \int_{-\pi}^{\pi} d t \\
& <\alpha(1-\rho) k+2[1-\alpha(1-\rho)] .
\end{aligned}
$$

Hence the result.

We note that $\mathrm{f}_{\alpha}$ is univalent for $\alpha<\frac{2}{(1-\rho)(k-2)}$, since $v_{m}$ consists of univalent functions for $2<m<4$. Hence $f_{\alpha}$ is univalent even if $f$ is not univalent provided $\alpha<\frac{2}{(1-\rho)(\overline{k-2})}$.

Using the standard technique, we can easily prove the following.

THEOREM 2.3. Let $g, h \in V_{k}(\rho)$ and let $\alpha>0, \beta>0$ and $\alpha+\beta<1-\rho$. Then

$$
H(z)=\int_{0}^{z}\left(g^{\prime}(t)\right)^{\alpha}\left(h^{\prime}(t)\right)^{\beta} d t
$$

is convex of order $\rho_{1}=\left(1-\frac{\alpha+\beta}{1-\rho}\right)$ for $|z|<r_{1}$,

where

$$
r_{1}=\frac{1}{2}\left[k-\sqrt{k^{2}-4}\right]
$$

The result is sharp when

$$
g^{\prime}(z)=h^{\prime}(z)=\left[\frac{(1-z)^{\left(\frac{k}{2}-1\right)(1-0)}}{(1+z)}\right] \text {. }
$$


We now prove the following.

THEOREM 2.4. Let $f: f(z)=z+\sum_{n=2}^{\infty} a_{n} z^{n} \varepsilon v_{k}(\rho)$. Then, for all $n>3$, $2<k<\infty$.

$$
\left|a_{n}\right|<\left[k^{2}(1-\rho)^{2}+k(1-\rho)\right] 2^{-2 \rho}\left(\frac{2 n}{3}\right)(1-\rho)\left(\frac{k}{2}+1\right)-2
$$

The function $f_{0}$ defined by (2.6) shows that the exponent $\left[(1-p)\left(\frac{k}{2}+1\right)-2\right]$ is best possible.

PROOF. By defintition, we have

Set

$$
\left(z f^{\prime}(z)\right)^{\prime}=f^{\prime}(z) p(z), \quad p \in P_{k}(\rho)
$$

$$
\begin{aligned}
F(z) & =\left(z\left(z f^{\prime}(z)\right)^{\prime}\right)^{\prime} \\
& =f^{\prime}(z)\left[p^{2}(z)+z p^{\prime}(z)\right] .
\end{aligned}
$$

For $z=r e^{i \theta}$, we have

$$
n^{3}\left|a_{n}\right|<\frac{1}{2 \pi r^{n-3}} \int_{0}^{2 \pi}\left|f^{\prime}(z)\right|\left|p^{2}(z)+z p^{\prime}(z)\right| d \theta
$$

Using (2.5) and theorem 1.3, we obtain

$$
\begin{aligned}
& n^{3}\left|a_{n}\right|<\frac{1}{r^{n-3}} \frac{(1+r)}{(1-\rho)\left(\frac{k-2}{2}\right)}{ }_{(1-\rho)\left(\frac{k+2}{2}\right)}^{(1-r)}\left\{\frac{1+k^{2}(1-\rho)^{2}-1 \mid r^{2}+k(1-\rho)}{1-r^{2}}\right\} \\
& =\frac{1}{r^{n-3}} \frac{(1+r)(1-\rho)\left(\frac{k-2}{2}\right)-1}{(1-r)}\left\{1+k(1-\rho)+\left\{k^{2}(1-\rho)^{2}-1\right\} r^{2}\right\}
\end{aligned}
$$

Let $r=1-\frac{3}{n}, n>3$. Then

$$
\begin{aligned}
n^{3}\left|a_{n}\right| & <\left[k^{2}(1-\rho)^{2}+k(1-\rho)\right] e^{3} \cdot\left(2-\frac{3}{n}\right)^{(1-\rho)\left(\frac{k-2}{2}\right)}\left(\frac{n}{3}\right)^{(1-\rho)\left(\frac{k+2}{2}\right)+1} \\
& =\left[k^{2}(1-\rho)^{2}+k(1-\rho)\right] e^{3} \cdot\left(\frac{n}{3}\right)^{\left[(1-\rho)\left(\frac{k+2}{2}\right)-2\right]} \cdot \frac{n}{27}^{3}\left(2-\frac{3}{n}\right)^{(1-\rho)\left(\frac{k}{2}-1\right)-1}
\end{aligned}
$$

Thus, for $n>3$, $\left|a_{n}\right|<\left[k^{2}(1-p)^{2}+k(1-\rho)\right](2)^{-2 \rho} \cdot\left(\frac{2 n}{3}\right)$

THEOREM 2.5. Let $f \in v_{k}(\rho)$, $\rho \neq 1 / 2$. Then $f$ maps $|z|<r_{0}$ onto a convex domain where $r_{0}$ is given by $(1.6)$. The function $f_{0}$, defined by $(2.6)$ shows that this result is sharp. 
The proof is straightforward and follows immediately from the definition and Theorem 1.5 .

Furthermore it can easily be shown that if $f \varepsilon V_{k}(\rho)$, then $f$ is convex of order $\rho$ for $|z|<r_{1}$ where $r_{1}$ is given by (2.8).

\section{THE CLASS $\mathbf{T}_{k}(\rho)$.}

A class $T_{k}$ of analytic functions related with the class $V_{k}$ has been introduced and studied in [5]. We now define the following.

DEFINITION 3.1. Let $r$ with $f(0)=0, f^{\prime}(0)=1$ be analytic in E. Then $f \varepsilon T_{k}(\rho), k>2,0<\rho<1$, if there exists a function $g \varepsilon V_{k}(\rho)$ such that $\frac{f^{\prime}(z)}{g^{\prime}(z)} \in P$ for $z \in E$.

Note that $T_{k}(0)=T_{k}$ and $T_{2}(0)$ is the class of close-to-convex functions.

THEOREM 3.1. Let $f \in T_{k}(n)$. Then

$$
|f(z)|>M_{2}(a+1, b ; c+1, r) \text {, }
$$

where $M_{2}(a, b ; c, r)$ is defined by $(2.2)$ and $a, b, c$ are given by $(2.4)$. This result is sharp.

PROOF. Since $f \in T_{k}(p)$, we can write

$$
f^{\prime}(z)=g^{\prime}(z) h(z), \quad g \in V_{k}(\rho), h \in P \text {. }
$$

It is we11-known that for $h \in P$

$$
|h(z)|>\frac{1-|z|}{1+|z|}
$$

Thus, using (3.1) and (2.5), we have

$$
\left|f^{\prime}(z)\right|>\frac{(1-|z|)^{\left(\frac{k}{2}-1\right)(1-\rho)+1}}{(1+|z|)^{\left(\frac{k}{2}+1\right)(1-\rho)+1}}
$$

Proceeding in the same way as in Theorem 2.1, we obtain the required result.

REMARK 3.1. When $\rho=0, f \varepsilon T_{k}$ and since in this case $b=0<1, c=1+a-b$, we have $G(a, b ; c,-1)=1$. Letting $r+1$, with $p=0$, in Theorem 3.1, we see that the image of $E$ under functions $f$ in $T_{k}$ constains the schlicht disk $|z|<\frac{1}{k+2}$.

We now give a necessary condition for a function $f$ to belong to the class $T_{k}(\rho)$.

THEOREM 3.2. Let $f \in T_{k}(\rho)$. Then, with $z=\operatorname{re}^{1 \theta}$ and $\theta_{1}<\theta_{2} ; 0<\rho<1$, 


$$
\int_{\theta_{1}}^{\theta_{2}} \operatorname{Re} \frac{\left(z f^{\prime}(z)\right)^{\prime}}{f^{\prime}(z)} d \theta>-k(1-\rho) \frac{\pi}{2}
$$

PROOF. We can write

$$
f^{\prime}(z)=\left(g_{i}(z)\right)^{1-\rho}\left(h_{1}(z)\right)^{1-p} \text {, for some } g_{1} \in V_{k}, h_{1} \in P \text {. }
$$

So

$$
f^{\prime}(z)=\left(g_{1}^{\prime}(z) h_{1}(z)\right)^{1-\rho}=\left(f_{1}^{\prime}(z)\right)^{1-\rho}
$$

for some $E_{1} \in T_{k}$.

Hence

$$
\frac{\left(z f^{\prime}(z)\right)^{\prime}}{f^{\prime}(z)}=(1-\rho) \frac{\left(z f_{i}^{\prime}(z)\right)^{\prime}}{f_{i}^{\prime}(z)}+\rho
$$

The required result follows on noting that, for $\theta_{1}<\theta_{2}, f_{1} \varepsilon T_{k}$

$$
\int_{0}^{\theta_{1}} \operatorname{Re} \frac{(\mathrm{zf} i(z))^{\prime}}{f_{i}^{\prime}(z)}-\mathrm{d} \theta>-\frac{k}{2} \pi, \text { see }[5] \text {. }
$$

REMARK 3.2. In [1], Goodman introduced the class $K(\beta)$ of normalized analytic functions which are close-to-convex of order $B>0$ and showed that if $f$ is analytic in $E$ and $f^{\prime}(z) \neq 0$, then for $\beta>0, f \in K(\beta)$ if for $z=\operatorname{re}^{i \theta}$ and $\theta_{1}<\theta_{2}$

$$
\int_{\theta}^{\theta} 2 \operatorname{Re} \frac{\left(z f^{\prime}(z)\right)^{\prime}}{f^{\prime}(z)} d \theta>-\beta \pi
$$

When $0<\beta<1, K(\beta)$ consists of univalent functions, whilst if $B>1$, $f$ need not even be finitely-valent.

We note that Theorem 3.2 shows that

$$
T_{k}(\rho) \subset K\left(\frac{k(1-\rho)}{2}\right) \text {. }
$$

Hence $T_{k}(\rho)$ consists entirely of univalent functions $1 f 2<k<\frac{2}{1-\rho}$. It also follows easily from the definition that the class $T_{k}(\rho)$ forms a subset of a linear-invariant family of order $\left[\frac{k}{2}(1-\rho)+1\right]$.

Using the method of Clunie and Pommerenke as modified by Thomas [7], we can easily prove the following:

THEOREM 3.3. Denote by $L(r, f)$ the length of the image of the circle $|u|=r$ under $f$ and by $M(r)=\max _{\theta}\left|f\left(r e^{i \theta}\right)\right|$. Then, for $0<r<1$,

$$
L(r)<A(k, \rho) M(r) \log \frac{1}{1-r}
$$

where $A(k, \rho)$ is a constant depending only on $k$ and $\rho$.

Let $P_{\alpha, 1}$ denote the class of functions $p(z)$ in $E$ given by 


$$
p(z)=1+c_{1} z+c_{2} z^{2}+\cdots \cdots \cdots \cdots \cdots
$$

which satisfy the inequality

$$
\left|p(z)-\frac{1}{2 \alpha}\right|<\frac{1}{2 \alpha}, 0<\alpha<1
$$

The class $P_{\alpha, 1}$ has been introduced in [8] and it is shown there that, for $P \& P_{\alpha, l},|z|=r<1$.

$$
\left|\frac{p^{\prime}(z)}{p(z)}\right|<\frac{(1+c)}{(1+c r)(1-r)} \text {, }
$$

where $\quad c=1-2 \alpha$

We now prove the following.

THEOREM 3.4. Let $g \in V_{k}(\rho)$ and let $\frac{f^{\prime}(z)}{g^{\prime}(z)} \in P_{\alpha, 1}$. Then $f$ is a convex function of order $\rho$ for $|z|<r$ where $r \varepsilon(0,1)$ is the least positive root of the equation

$(1-\rho) c x^{3}-[(\rho+c)+c k(1-\rho)] x^{2}+[\rho(k-c)-(1+k)] x+(1-\rho)=0$

PROOF. We can write

$$
f^{\prime}(z)=\left(g_{1}^{\prime}(z)\right)^{1-\rho} p(z), g_{1} \varepsilon V_{k}, \quad p \in P_{\alpha, 1}
$$

So

$$
\left.\operatorname{Re}\left[\frac{\left(z f^{\prime}(z)\right)^{\prime}}{f^{\prime}(z)}-p\right]>(1-p) \operatorname{Re}\left[\frac{\left(z g_{1}^{\prime}(z)\right)^{\prime}}{g_{1}^{\prime}(z)}\right]-\frac{z p^{\prime}(z)}{p(z)}\right]
$$

Using Theorem 1.4 with $\rho=0$ and $(3.4)$, we have the required result.

Furthermore, if

$T(r)=(1-\rho) c r^{3}-[(\rho+c)+c k(1-\rho)] r^{2}+[\rho(k-c)-(1+k)] r+(1-\rho)$,

then we note that

$$
\begin{aligned}
& T(0)=(1-\rho)>0 \\
& T(1)=-2 \rho c-2 \rho-c k(1-\rho)-k(1-\rho)<0
\end{aligned}
$$

Thus $r \in(0,1)$.

COROLLARY 3.1. When $\alpha=0, c=1$ and $\rho=0, f \in T_{k}$. Thus $f$ maps $|z|<r=\frac{1}{2}\left[(k+2)-\sqrt{k^{2}}+4 k\right]$ onto a convex domain and this result is sharp, see $[5]$.

COROLLARY 3.2. When $\rho=0, \alpha=\frac{1}{2}$, and then we have $\left|\frac{f^{\prime}(z)}{g^{\prime}(z 0}-1\right|<1$ for $g \varepsilon V_{k}$. Then $f$ is convex for $|z|<r=\frac{1}{k+1}$. For $k=4, V_{k}$ consists of univalent functions and in this case $r=\frac{1}{5}$. This result is proved in [8]. For $\alpha=0, k=4$ and $\rho=0$, we obtain the known result $r=3-2 \sqrt{2}$ of Ratti [9] and when $k=2$, we have the wel1-known result giving us the radius of convexity for close-to-convex functions. 
Finally we have

THEOREM 3.5. Let $f \in V_{k}(\rho)$ and let

$$
F(z)=\frac{1}{1+m} z^{1-m}\left|z^{m} f(z)\right| ', \quad m=1,2,3, \ldots \ldots \ldots .
$$

Then $F \in T_{k}(\rho)$ for all $|z|<r_{2}$, where, for $(1-2 p-m) \neq 0,0<p<1$,

$$
r_{2}=2(1+m) /\left[(1-p) k+\sqrt{(1-p)^{2} k^{2}-4(1-2 p-m)(1+m)}\right]
$$

The proof is straightforward when we note that

$$
\operatorname{Re} \frac{F^{\prime}(z)}{f^{\prime}(z)}=\frac{1}{1+m}\left[\left\{\operatorname{Re} \frac{\left(z f^{\prime}(z)\right)^{\prime}}{f^{\prime}(z)}\right\}+m\right]
$$

and then use theorem 1.4 .

ACKNOWLEDGEMENT. The author is grateful to the referee for his valuable comments and suggestions.

\section{REFERENCES}

1. PadmanabHan, K.S. and Parvatham, R. 'Properties of a class of functions with bounded boundary rotation', Ann. Polon. Math. 31(1975), 311-323.

2. PINCHUK, B. Functions with bounded boundary rotation, I.J. Math. $10(1971), 7-16$.

3. GOODMAN, A.W. Univalent Functions, Vol. I, II, Mariner Publishing Company, Tempa, Florida, U.S.A. (1982).

4. WHITTAKER, E. and WATSON, G. A course of modern analysis, Cambridge Univ. Press, New York, 1927.

5. NOOR, K.I. On a generalization of close-to-convexity, Int. J. Math. \& Math. Sc1. $6(1983), 327-334$.

6. GOODMAN, A.W. On close-to-convex functions of higher order, Ann. Univ. Sci. Budapest Eötöus Sect. Math. 25(1972), 17-30.

7. THOMAS, D.K. On close-to-convex functions, Publ. Inst1t. Math. $\underline{45}(1989), 85-88$.

8. SHAFFER, D.B. Radii of starlikeness and convexity for special classes of analytic functions, J. Math. Analysis and Appl. 45 $(1974), 73-80$.

9. RATTI, J.S. The radius of convexity of certain analytic functions, Indian J. Pure Appl. Math. 1(1970), 30-37. 


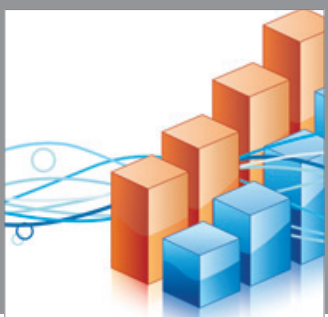

Advances in

Operations Research

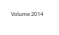

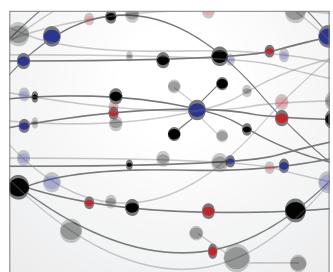

\section{The Scientific} World Journal
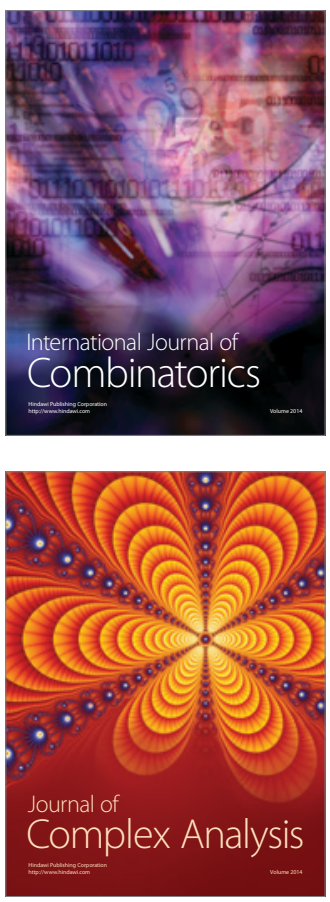

International Journal of

Mathematics and

Mathematical

Sciences
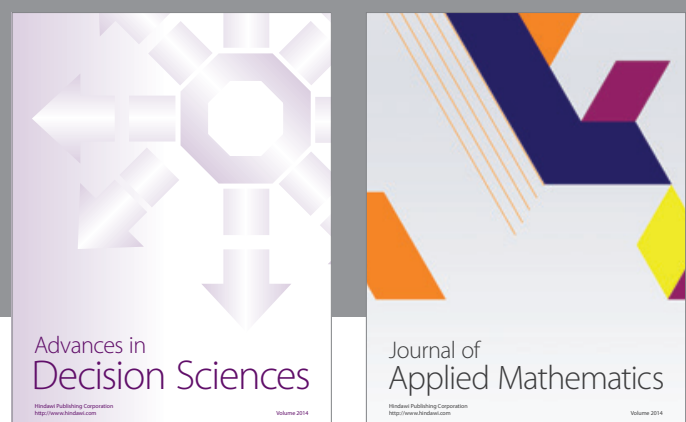

Journal of

Applied Mathematics
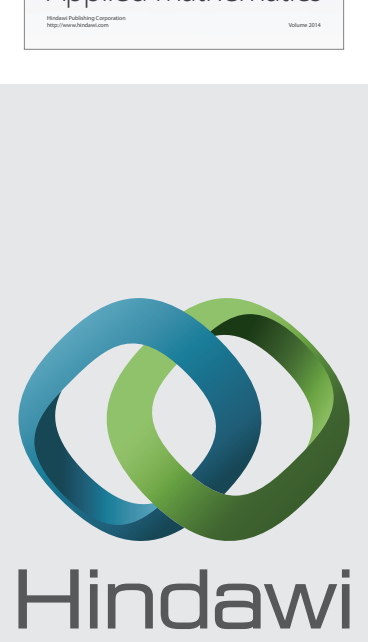

Submit your manuscripts at http://www.hindawi.com
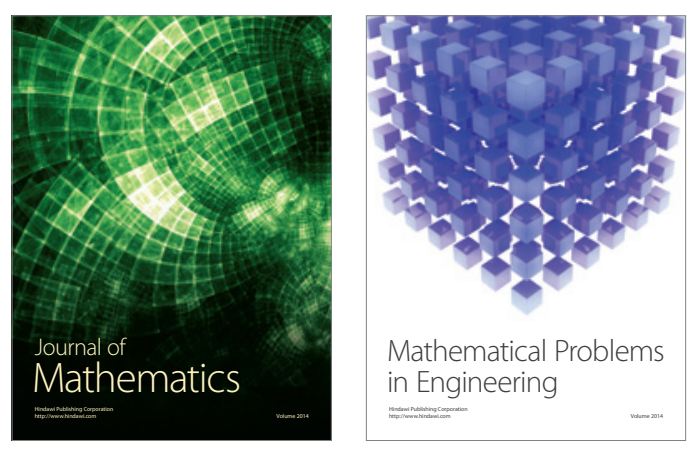

Mathematical Problems in Engineering
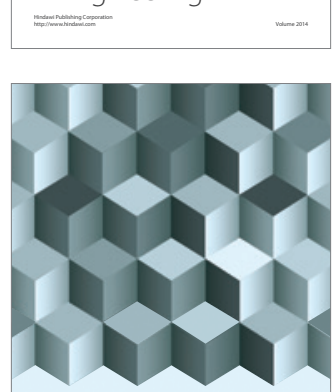

Journal of

Function Spaces
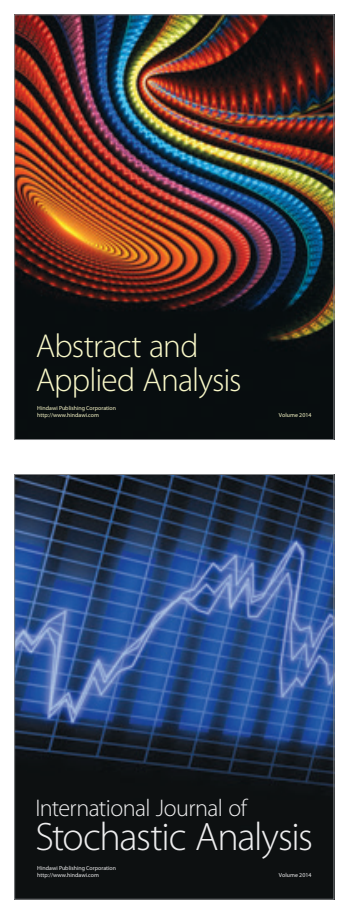

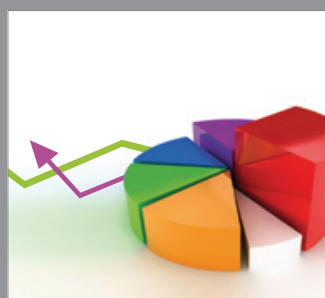

ournal of

Probability and Statistics

Promensencen
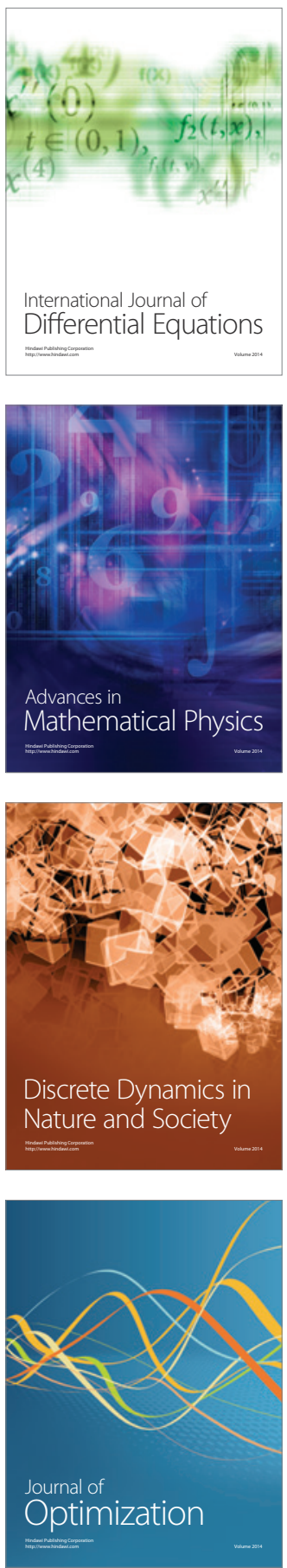